\title{
Reduktion prooxidativer und Stärkung antioxidativer Prozesse - ein verbindendes Element der Kneipp'schen Therapie
}

\author{
Rainer Brenke $e^{a, b} \quad$ Werner Siems ${ }^{b}$ \\ aBerlin,

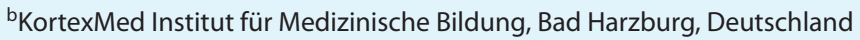

\section{Schlüsselwörter \\ Kneipp-Therapie · Naturheilverfahren · Oxidativer Stress . \\ Freie Radikale · Antioxidative Protektion}

\section{Zusammenfassung}

Die Kneipp'schen Therapieverfahren und damit zentrale Inhalte der klassischen Naturheilverfahren zeichnen sich durch ein breites Wirkungsspektrum aus. Es ist naheliegend, dass diesen Verfahren auch gemeinsame Wirkungsmechanismen auf der Basis ubiquitärer Substanzen und Reaktionsmechanismen zugrunde liegen. Hier wurden die Wirkungen von reaktiven Sauerstoffspezies (ROS), von Lipidperoxidations(LPO)-Produkten und von antioxidativen Schutzmechanismen untersucht. Die Bilanz von prooxidativen und antioxidativen Prozessen, die das Mass für einen oxidativen Stress angibt, hat eine immense Auswirkung auf Gesundheit, Krankheit, Wohlbefinden und Leistungsfähigkeit. Die Übersicht über Resultate zu allen fünf Säulen der KneippTherapie - Hydrotherapie, Phytotherapie, Ernährungstherapie, Bewegungstherapie und Ordnungstherapie - liefert klare Hinweise auf die bessere Bewältigung von oxidativem Stress durch die Kneipp'schen Therapieformen. Es wird deutlich aufgezeigt, dass eine verminderte Bildung freier Radikale und/oder deren bessere Bewältigung ein verbindendes Element der klassischen Naturheilverfahren ist.

\section{Keywords}

Kneipp therapy · Naturopathy · Oxidative stress · Free radicals .

Antioxidative protection

\section{Summary}

Reduction of Prooxidative and Strengthening of Antioxidative Processes a Combining Element in Kneipp Therapy

The aspects of Kneipp therapy and of classical natural medicine are characterized by a wide spectrum of effects and efficacy. We suggest that the basic mechanisms of such classical types of therapy are elementary and unique being based on ubiquitous substances and biochemical reactions. In this regard, we investigated the reactive oxygen species (ROS), lipid peroxidation (LPO) products, and antioxidative protective mechanisms. The balance between prooxidative and antioxidative mechanisms - characterizing the so-called oxidative stress - exerts high impact on the status of health, disease, wellbeing, and fitness. The review on all five parts of Kneipp therapy - hydrotherapy, phytotherapy, nutritional therapy, exercise therapy, and regulatory therapy - supplies distinct arguments for improved coping with oxidative stress by means of all forms of Kneipp therapy. It is clearly demonstrated that the reduced formation of free oxygen radicals and/or the increased removal of these potentially toxic substances is a combining element in natural medicine.

\section{Mots-clés}

Thérapie selon Kneipp · Médecine naturelle · Stress oxydant . Radicaux libres · Protection anti-oxydante

\section{Résumé}

Réduction du processus pro-oxydant et renforcement du processus anti-oxydant: élément unifiant de la thérapie selon Kneipp

La méthode thérapeutique selon Kneipp et les éléments centraux de la médecine naturelle classique se caractérisent par un vaste spectre d'action. II est tout naturel que même des mécanismes d'action communs reposant sur des substances ubiquitaires et des mécanismes de réaction soient à la base de cette méthode. On a étudié ici les effets des dérivés réactifs de l'oxygène (DRO), des produits de lipidoperoxydation (LPO) et des mécanismes anti-oxydants de protection. Le bilan des processus pro-oxydants et anti-oxydants qui donne la mesure d'un stress oxydant exerce un énorme effet sur la santé, la maladie, le bienêtre et les performances. L'aperçu des résultats de l'analyse des cinq piliers de la thérapie selon Kneipp (hydrothérapie, phytothérapie,

\section{KARGER \\ Fax +497614520714 Information@Karger.com} www.karger.com
PD Dr. Rainer Brenke

Hermsdorfer Strasse 31, 13469 Berlin, Deutschland DrBrenke@aol.com 
thérapie alimentaire, thérapie motrice et thérapie de l'ordre) donne des indications claires sur la meilleure façon de combattre le stress oxydant en appliquant les différentes formes de thérapies selon Kneipp. II est nettement démontré qu'une formation réduite de radicaux libres et/ ou une meilleure prise en compte de ces derniers constituent un élément unifiant de la médecine naturelle classique.

\section{Einleitung}

"Ich trage kein innigeres Verlangen und wünsche, als dass endlich die Leute vom Fach, die Herren Ärzte, allgemeiner und umfassender auch die Wasserheilmethode gründlich studieren und in die Hand und Aufsicht nehmen mögen.» (Sebastian Kneipp)

Zu den klassischen Kneipp'schen Therapieverfahren zählen die Hydrotherapie, die Phytotherapie, die Ernährungstherapie, die Bewegungstherapie und die Ordnungstherapie. Diese Verfahren sind in der Medizin allgemein etabliert; ihre Wirksamkeit für die Prophylaxe und Therapie, für Gesundheit und Leistungsfähigkeit ist weitgehend unbestritten. Dennoch ist es für eine weitere Verbreitung des Kneipp'schen Gedankengutes unerlässlich, zugrunde liegende physiologische, biochemische und immunologische Mechanismen weiter zu klären. Wegen des grossen Indikationsspektrums und der therapeutischen Breite dieser Verfahren liegt die Vermutung nahe, dass die Bestandteile der Kneipp'schen Therapie auch ein allgemeines Wirkprinzip auf biochemischer Ebene haben. Ein solches grundlegendes Wirkprinzip dürfte auf ubiquitären Substanzen und ubiquitären chemischen und biochemischen Reaktionen beruhen. Dafür kommen nur wenige Substanzen und deren Reaktionen infrage. Wenn es um generalisierte Fragen von Gesundheit und Krankheit geht, wird es sich auch um Reaktionen und Metabolite von Substanzen handeln, die regelmässig und in ausreichender Konzentration auf den Organismus einwirken. Wir denken dabei an Glukose, Cholesterol, Sauerstoff oder Wasser. In dieser Arbeit werden die Wirkungen von reaktiven Sauerstoffspezies (ROS), also von Produkten des Sauerstoffs wie z.B. von Lipidperoxidations(LPO)-Produkten, und von Schutzmechanismen gegen die Reaktionen der ROS, die man antioxidative Schutzmechanismen nennt, untersucht. Die Bilanz von prooxidativen und antioxidativen Prozessen, die das Mass für einen oxidativen Stress angibt, hat eine immense Auswirkung auf Gesundheit, Krankheit, Wohlbefinden und Leistungsfähigkeit. Es gibt eine grosse Anzahl von Erkrankungen, in deren Pathogenese oxidativer Stress bedeutsam ist (Tab. 1) [1-22]. Darüber hinaus hat der oxidative Stress entscheidenden Einfluss auf das Ausmass und die Geschwindigkeit von Alterungsprozessen [23-28]. Anliegen der vorliegenden Übersicht ist es, Prozesse der Bildung sogenannter freier Radikale und der
Aktivität antioxidativer Schutzsysteme, d.h. letzten Endes den oxidativen Stress, im ZusammenhangmitKneipp'schen Therapieverfahren zu betrachten.

Als freie Radikale bezeichnet man Atome oder Moleküle mit einem oder mehreren ungepaarten Elektronen. Häufig besitzen diese eine hohe Reaktivität. Da Sauerstoff $\left(\mathrm{O}_{2}\right)$ ubiquitär auftritt und seine permanente Zufuhr für den Menschen unerlässlich ist, sind im menschlichen Organismus Sauerstoffradikale/ROS besonders verbreitet. Sie sind in der Lage, prooxidative Wirkungen auszuüben. Zum Schutz vor freien Radikalen und anderen ROS hat der Körper mannigfaltige Schutzmechanismen entwickelt, die man unter dem Begriff der antioxidativen Schutzmechanismen zusammenfasst [29].

Die Janusköpfigkeit des Sauerstoffs besteht in seinem Nutzen zur Energiegewinnung und seinem potenziellen Schaden durch ROS. Andererseits besteht auch ein Wechselspiel zwischen ROS und antioxidativen Schutzmechanismen. Zum einen ist die Bildung freier Radikale mit dem Ablauf wichtiger physiologischer Reaktionen in aerob lebenden Organismen untrennbar verbunden [30]. So spielen freie Radikale bei der Phagozytose, bei der Pyruvatoxidation und in anderen enzymatischen Reaktionen eine wichtige Rolle und sind damit für das Leben unverzichtbar. Bei einem Übermass von ROS geht man jedoch davon aus, dass Krankheiten entstehen und Alterungsprozesse beschleunigt werden können (Tab. 1).

Heute gelten freie Radikale und ROS überhaupt als eine mögliche Ursache für die Alterung von Zellen [23-28, 31]. In den letzten Jahren schien diese Theorie durch die Telomerenverkürzung der Chromosomen verdrängt zu werden. Man konnte beobachten, dass Telomeren sich mit zunehmender Häufigkeit der Zellteilungen und daher mit zunehmendem Alter verkürzen, und hat vermutet, dass dies im Zusammenhang mit dem genetischen Alterungsprozess steht. Doch wie hoch ist die Zahl der Zellteilungen? Genetiker schätzen, dass eine Körperzelle im Durchschnitt der 50. bis 60. Nachkomme der Eizelle ist, aus der sie entstanden ist, wenn auch die Häufigkeit der Teilung nicht in allen Teilen des wachsenden Organismus gleich ist. Es ist jedoch leicht einschätzbar, dass durch das rasche Anwachsen der Zellzahl 50-60 aufeinanderfolgende Zellteilungen ausreichen, um den Makroorganismus aus der Startzelle zu formen. Der gesamte Zellpool eines Menschen wird dann «nach einer sehr rohen Schätzung etwa 100 Billionen bzw. 1 Trillion» Zellen geschätzt, wie Erwin Schrödinger in «Was ist Leben?» auf der Grundlage von 1933 in Dublin gehaltenen Vorlesungen schreibt [32]. Bei den Teilungen läuft die Telomerenverkürzung ab. Neueste Forschungsergebnisse haben gezeigt, dass freie Radikale am Prozess der Verkürzung der Telomeren massgeblich beteiligt sind. Man nimmt an, dass die Telomeren als eine Art Sensor für schädigende Noxen aus der Umwelt angesehen werden können. Durch freie Radikale 
Tab. 1. Krankheiten, in deren Pathogenese oxidativer Stress nachgewiesen wurde

\begin{tabular}{ll}
\hline Krankheit & Wichtige Faktoren für den oxidativen Stress \\
\hline Arteriosklerose & Akkumulation von LDL in den Plaques, Anstieg von Protein- \\
& carbonylen und von LPO-Produkten wie MDA und HNE, auch \\
& von Oxisterolen im Blutplasma und in den Plaques, Entzündungs- \\
& prozesse in der Intima
\end{tabular}

Krebs

Rolle von ROS bei der Veränderung des genetischen Materials

(Cancerogenes), zum Teil verringerte DNA-Reparatur, erhöhte mitochondriale ROS-Bildung

Literatur

Siems et al., 2005 [1]

Spickett et al., 2010 [2]

Negre-Salvayre et al., 2010 [3]

Poli et al., 2008 [4]

Negre-Salvayre et al., 2010 [3]

Poli et al., 2008 [4]

Gueraud et al., 2010 [5]

Zarkovic et al., 2005 [6]

Ray et al., 2012 [7]

Grune et al., 1994 [8]

Infarkte (Ischämie und Reoxigenierung,
z.B. Herz, Gehirn)

Entzündungen, Infektionen

Diabetes mellitus

Rheumatoide Arthritis, unter anderem

Erkrankungen des rheumatischen

Formenkreises

\begin{tabular}{|c|c|c|}
\hline Strahlenbedingte Schäden & $\begin{array}{l}\text { Bildung von } \mathrm{H}_{2} \mathrm{O}_{2} \text {, unter anderem ROS durch ionisierende } \\
\text { Strahlung, Röntgenstrahlen, UV-Strahlung }\end{array}$ & $\begin{array}{l}\text { Siems et al., } 2005[1] \\
\text { Spickett et al., } 2010 \text { [2] } \\
\text { Poli et al., } 2008[4]\end{array}$ \\
\hline $\begin{array}{l}\text { Neurodegenerative Erkrankungen } \\
\text { (Multiple Sklerose, Amyotrophe } \\
\text { Lateralsklerose, Morbus Alzheimer usw.) }\end{array}$ & $\begin{array}{l}\text { Verminderung antioxidativer Schutzmechanismen, teilweise } \\
\text { auch verminderter proteasomaler Abbau oxidativ modifizierter } \\
\text { Proteine, zum Teil Autooxidation von Katecholaminen }\end{array}$ & $\begin{array}{l}\text { Spickett et al., } 2010 \text { [2] } \\
\text { Negre-Salvayre et al., } 2010[3] \\
\text { Chen et al., 2011 [13] } \\
\text { Castellani et al., 2012 [14] }\end{array}$ \\
\hline $\begin{array}{l}\text { Katarakt, altersbedingte } \\
\text { Makuladegeneration (AMD) }\end{array}$ & $\begin{array}{l}\text { ROS-bedingte Proteindenaturierung und -vernetzung, strahlen- } \\
\text { bedingte Degeneration von Photorezeptoren der Macula lutea }\end{array}$ & $\begin{array}{l}\text { Negre-Salvayre et al., } 2010[3] \\
\text { Poli et al., } 2008[4] \\
\text { Fletcher, } 2010[15]\end{array}$ \\
\hline Medikamenteninduzierte Schädigungen & $\begin{array}{l}\text { Xenobiotika-Entgiftung mit Cytochrom P450-assoziierter } \\
\text { ROS-Bildung }\end{array}$ & $\begin{array}{l}\text { Siems et al., } 2005[1] \\
\text { Poli et al., } 2008[4]\end{array}$ \\
\hline Chronische Niereninsuffizienz & $\begin{array}{l}\text { verringerte antioxidative Schutzsysteme der Erythrozyten als } \\
\text { mobile Radikalfänger bei kardiorenalem Anämiesyndrom, } \\
\text { Anstieg von HNE, MDA, Proteincarbonylen, wahrscheinlich } \\
\text { verstärkte Eisen-induzierte ROS-Bildung }\end{array}$ & $\begin{array}{l}\text { Spickett et al., } 2010 \text { [2] } \\
\text { Negre-Salvayre et al., } 2010[3] \\
\text { Poli et al., } 2008 \text { [4] } \\
\text { Wiswedel et al., } 2008 \text { [16] } \\
\text { Siems et al., 2005 [17] } \\
\text { Siems et al., } 2003 \text { [18] } \\
\end{array}$ \\
\hline Lymphödem & $\begin{array}{l}\text { erhöhte Granulozytenaktivität im lymphödematösen Gewebe } \\
\text { (NADPH-Oxidase, Myeloperoxidase), regionale Ischämie- } \\
\text { Reperfusionsprozesse mit verstärktem Purinabbau, GSH-Mangel }\end{array}$ & $\begin{array}{l}\text { Negre-Salvayre et al., } 2010[3] \\
\text { Poli et al., } 2008[4] \\
\text { Siems et al., 2002 [19] } \\
\text { Siems und Brenke, 2004 [20] } \\
\text { Brenke und Siems, } 2005 \text { [21] } \\
\text { Siems et al., 2009 [22] }\end{array}$ \\
\hline
\end{tabular}

geschädigte Zellen entarten zudem leichter. Bei einem Übermass an Belastungen stirbt die Zelle oft durch den Radikal-vermittelten Prozess des programmierten Zelltodes (Apoptose) ab. Dadurch wird die Gefahr einer malignen Entartung der Zelle geringer bzw. bereits mutierte Zellen vernichten sich im Interesse des Gesamtorganismus selbst. Auf jeden Fall hat oxidativer Stress Einfluss auf die Telomeren, und die Auffassungen von der Telomerenverkürzung stehen nicht im Widerspruch zur Radikaltheorie des Alterns, die vor mehr als 50 Jahren von Dan Harman postuliert wurde. Dass sich mit höherem Alter beim Menschen Produkte des oxidativen Stresses anhäufen, konnten wir mit altersabhängigen Messungen im Blutplasma und in Erythrozyten belegen [24, 25]. Dass sich im Laufe des Lebens und der Alterung die Anteile von ROS-Bildung und ROS-Beseitigung verändern - zuungunsten der antioxidativen Schutzmechanismen -, haben wir bei Beobachtungen an Ratten erkannt [26]. Ausserdem haben wir hierfür Erkenntnisse an menschlichen Fibroblasten gewonnen, bei denen im Verlaufe des Alterns einige Wege für den Abbau des LPO-Produktes HNE (Hydroxynonenal) langsamer werden [33]. 
Mithilfe Kneipp'scher Anwendungen könnte man - soweit sie Einfluss auf das Ausmass des oxidativen Stresses sowie auf die Balance zwischen prooxidativen und antioxidativen Prozessen haben - möglicherweise auch Geschwindigkeit und Ausmass von Alterungsprozessen beeinflussen. Auf jeden Fall könnte es auf diese Weise möglich sein, unphysiologisch beschleunigte prooxidative Alterungsprozesse zu reduzieren.

Ausserdem spielen freie Radikale bei einer Vielzahl von Erkrankungen eine Rolle in der Pathogenese, so z.B. bei rheumatischen Erkrankungen, anderen Entzündungsprozessen, der Tumorentstehung, der Arteriosklerose, dem Herzinfarkt, dem Schlaganfall, dem Diabetes mellitus, strahlenbedingten Erkrankungen der Haut und dem Katarakt [4] (Tab. 1).

Erst dann entstehen Schäden, wenn bei der Entstehung von freien Radikalen die antioxidativen Mechanismen in ihrer Kapazität überbeansprucht werden. Natürliche Antioxidantien sind z.B. Vitamin C, Vitamin E und das reduzierte Glutathion (GSH). Diese Substanzen und Enzyme, wie die Superoxiddismutase (SOD), die Glutathionperoxidase, die Glutathionreduktase sowie Enzyme der NADPH-Regeneration, werden zu den primären antioxidativen Schutzsystemen gerechnet. Die enzymatischen und nichtenzymatischen antioxidativen Schutzsysteme zeichnen sich durch ihr ubiquitäres Vorkommen und durch die Vielzahl von physiologischen und pathologischen Prozessen, an denen sie beteiligt sind, aus [34-36]. Sekundäre antioxidative Prozesse (Detoxifikationsphase II) tragen zur Entgiftung von sekundären toxischen Verbindungen bei. Dazu gehört z.B. der Abbau oxidativ modifizierter Eiweisse und der Metabolismus aldehydischer LPO-Produkte wie HNE und Malondialdehyd (MDA) [4, 33, 34, 37-42] (Abb. 1).

\section{Primärer antioxidativer Schutz}

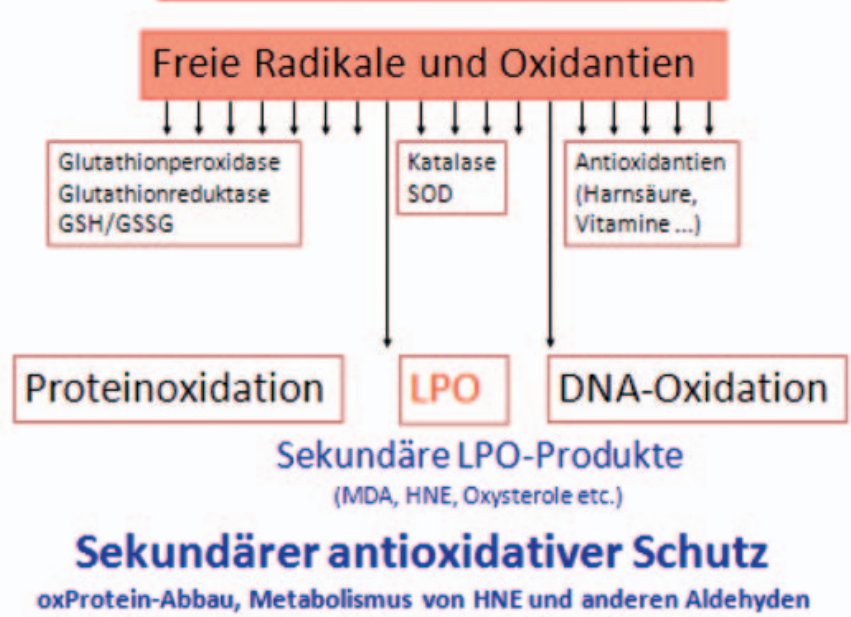

Abb. 1. Antioxidative Schutzmechanismen im Organismus.
Kneipp'sche Therapieverfahren sind typisch für Methoden einer ganzheitlichen Therapie. Sie behandeln nicht nur einen erkrankten Körperteil, sondern stets den ganzen Menschen. Es ist daher naheliegend, dass Verfahren mit einem breiten Indikationsspektrum auch über allgemein verbreitete molekulare Mechanismen wie die der Bildung und Bewältigung von ROS wirksam werden. In diesem Zusammenhang ist es von Bedeutung, dass einem allgemein verbreiteten Mangel an natürlichen Reizen (thermische Reize, Bewegung, gesunde Ernährung) ein Übermass an Stress auf psychologischer Ebene gegenübersteht. Man versucht, fehlende natürliche Reize durch präventive oder therapeutische Reize zu kompensieren. Dazu gehören viele Verfahren der Hydrotherapie, ein dosiertes körperliches Training, eine gesunde Vollwerternährung, Stressabbau, ein geordneter Tagesablauf usw.

Im Folgenden werden Ergebnisse aus unserer Arbeitsgruppe dargestellt, die zunächst auf dem Gebiet der Hydrotherapie gewonnen wurden. Die hier erhaltenen Ergebnisse ermutigten uns, auch weiterführende Untersuchungen auf dem Gebiet der Phytotherapie, der Ernährungstherapie (hier insbesondere im Zusammenhang mit dem therapeutischen Fasten) sowie der Bewegungstherapie und auch der Ordnungstherapie vorzunehmen. Die folgende Übersicht enthält im Wesentlichen eine Zusammenfassung der Ergebnisse eigener Untersuchungen zu diesem Thema aus den letzten zwei Jahrzehnten, was den Vorteil hat, dass sie mit einheitlicher Methodik gewonnen wurden und daher unmittelbar vergleichbar sind. Wichtige Arbeiten anderer Autoren wurden berücksichtigt. Die Autoren hoffen, dass die Untersuchungen nicht nur zu einer weiteren wissenschaftlichen Anerkennung der Kneipp'schen Therapieverfahren führen, sondern vor allem auch zu deren weiterer Propagierung beitragen.

Abbildung 2 versinnbildlicht die fünf Säulen der Kneipp'schen Therapie mit dem Sauerstoff und den von ihm gebildeten freien Radikalen im Wechselspiel vom oxidativen Substrat zur Energiegewinnung und vom Präkursor der aggressiven Sauerstoffradikale.

Abb. 2. Bildung und Bewältigung freier Radikale ein gemeinsames Element der klassischen Naturheilverfahren.

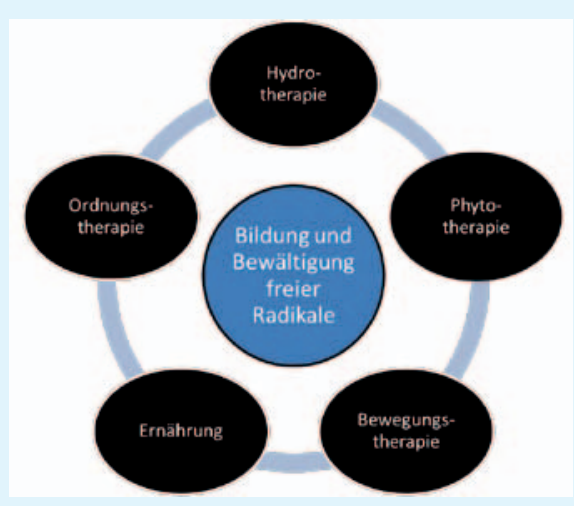




\section{Hydrotherapie}

"Es leuchtet ein, dass in einem jeden Krankheitsfalle verschiedene Wasseranwendungen vorkommen müssen, ferner dass nicht der kranke Körperteil allein in Behandlung kommt, sondern stets der ganze Körper.» "Dreimal - ich gestehe es offen - sah ich mich veranlasst, meine Wasserverfahren zu ändern, die Saiten abzuspannen - von der Strenge zur Milde, von grosser Milde zu noch grösserer herabzusteigen.»

(Sebastian Kneipp)

Die Hydrotherapie stellt die Hauptsäule der Kneipp'schen Therapieverfahren dar. Speziell der Begriff der Abhärtung wird mit der wiederholten Anwendung von Kaltreizen identifiziert. Allgemein verstehen wir unter Abhärtung die wiederholte bewusste oder unbewusste Exposition des Menschen gegenüber natürlichen Reizen mit der Folge einer Erhöhung der Widerstandsfähigkeit gegenüber Krankheiten, vor allem gegenüber grippalen Infekten und Entzündungen des Respirationstrakts. Die Abhärtung durch Hydrotherapie beruht auf der Beeinflussung unterschiedlicher Regulationsmechanismen [43]. Viele molekulare Feinmechanismen der Abhärtung durch Kaltreize sind noch nicht geklärt. Wir haben daher eine Methode, bei der sich die Probanden freiwillig einem besonders intensiven Kaltreiz aussetzen, näher untersucht. Diese durch uns untersuchte besonders extreme Form der Abhärtung stellt das Winterschwimmen oder Eisbaden dar [44, 45] (Abb. 3).

Ausgangspunkt für unsere Untersuchungen zur Wirkung von Kaltreizen war die Beobachtung, dass es beim Winterschwimmen zu einem schnellen Abfall der Harnsäure-Konzentration im Blutplasma kommt. Dieser Abfall ist dosisabhängig - in geringerem Umfang kann man ihn auch bei kaltem Duschen beobachten [46-50]. Es konnte gezeigt werden, dass der Abfall der HarnsäureKonzentration nicht durch gesteigerte Ausscheidung oder Hemmung der Synthese der Harnsäure erklärt werden kann, sondern dass er mit der Funktion der Harnsäure als Fänger freier Radikale, insbesondere der hochreaktiven Hydroxylradikale, zusammenhängt [51, 52]. Weitere Untersuchungen zeigten, dass es neben dem Abfall der Harnsäure-Konzentration auch zu deutlichen Veränderungen des Glutathionstatus in den Erythrozyten kommt [51, 52], dem ebenfalls eine wichtige Rolle als antioxidatives Schutzsystem zukommt $[35,36]$. Nach dem Eisbad ist die Konzentration des GSH verringert und die Konzentration der oxidierten Form des Glutathions (GSSG) erhöht (Abb. 4). Dies spricht für die oxidative Belastung während des Winterschwimmens. Die Hauptquelle für die verstärkte Radikalbildung beim Winterschwimmen ist die mitochondriale Atmungskette der Skelettmuskelzellen. Besonders interessant war die Tatsache, dass langfristig An-

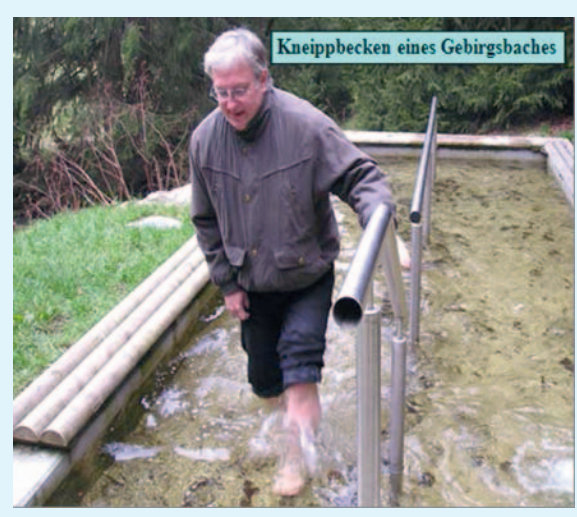

Abb. 3. Hydrotherapie: Einer der Autoren beim Wassertreten im Erzgebirge.

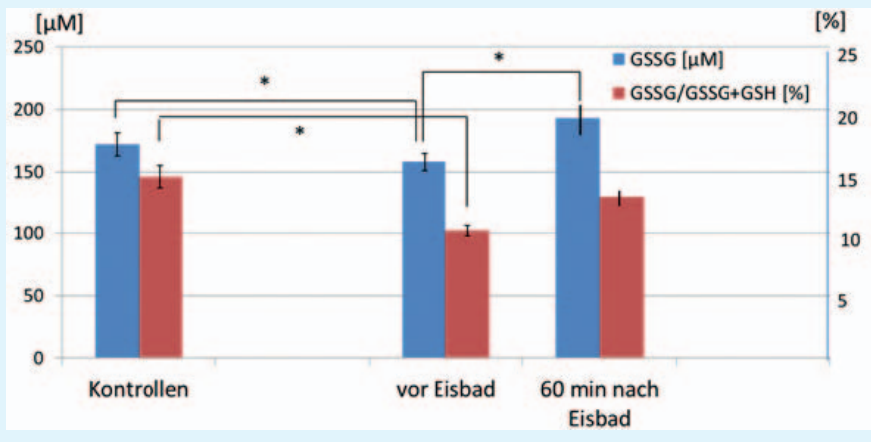

Abb. 4. Konzentration der oxidierten Form des Glutathions (GSSG) und Verhältnis von GSSG und Gesamt-Glutathion (\%) in Erythrozyten bei Kontrollpersonen sowie Winterschwimmern vor und 60 min nach Eisbad ( $\mathrm{n}=10$, Mittelwerte und Standardfehler). Es besteht ein signifikanter Unterschied $\left(^{*}\right)$ bei GSSG sowie beim Quotienten GSSG/Gesamt-Glutathion zwischen den Kontrollpersonen und den Ausgangswerten der Winterschwimmer ( $<<0,05$; Wilcoxon-Test). Ebenso besteht ein signifikanter Anstieg $(\mathrm{p}<0,05)$ des GSSG bei den Winterschwimmern 60 min nach dem Eisbad im Vergleich zu ihren Ausgangswerten vor dem Eisbad.

passungserscheinungen im Sinne einer Erhöhung des Gesamt-Glutathiongehalts und vor allem des Gehalts an der antioxidativ wirksamen Komponente des GSH der Erythrozyten der Winterschwimmer auftreten [52, 53]. Der intraerythrozytäre Gehalt des GSSG ist bei den Winterschwimmern im Vergleich zu Kontrollpersonen vermindert (Abb. 4). Man kann also davon ausgehen, dass der Organismus durch wiederholte Kaltreize und damit eine dosierte oxidative Belastung in die Lage versetzt wird, im Bedarfsfall besser freie Radikale zu bewältigen. Neben Anpassungen im Glutathionsystem sieht man nach wiederholten Kaltreizen oder Wechselreizen in der Tat deutliche Veränderungen bei verschiedenen antioxidativen Schutzenzymen. Die Aktivitäten der SOD und der Katalase sind bei Personen, die sich regelmässig Kaltreizen aussetzen, im Gegensatz zu Vergleichspersonen deutlich erhöht [53].

Anhand mehrerer klinischer und Laborparameter wurde regelmässig kontrolliert und nachgewiesen, dass es zu keinerlei gesundheitlicher Schädigung der Eisbader gekommen ist. 
Im Jahr 2011 wurden in mehreren Arbeiten in verschiedenen Geweben beim Menschen sowie unter anderem bei Wirbeltieren Befunde erhoben, die den oxidativen Stress nach Ganzkörper-Kaltreizen beweisen und ebenso die antioxidative Adaptation an regelmässig wiederholte nicht schädigende Kaltreize dokumentieren [54-56]. Wir sehen in diesen Befunden eine eindrucksvolle Bestätigung unserer Erkenntnisse bei Winterschwimmern. Seit geraumer Zeit propagieren wir das Regimen refrigerans erfolgreich und wissenschaftlich untermauert im Rahmen der Prävention von Infekten [57, 58].

Für die Praxis ist es relevant, dass auch die Sauna mit ihren thermischen Wechselreizen zu einer akuten radikalischen Belastung des Organismus führt, wenngleich selbige deutlich schwächer ausfällt als bei den Winterschwimmern [59]. Dennoch sind hier ähnliche Wirkungen

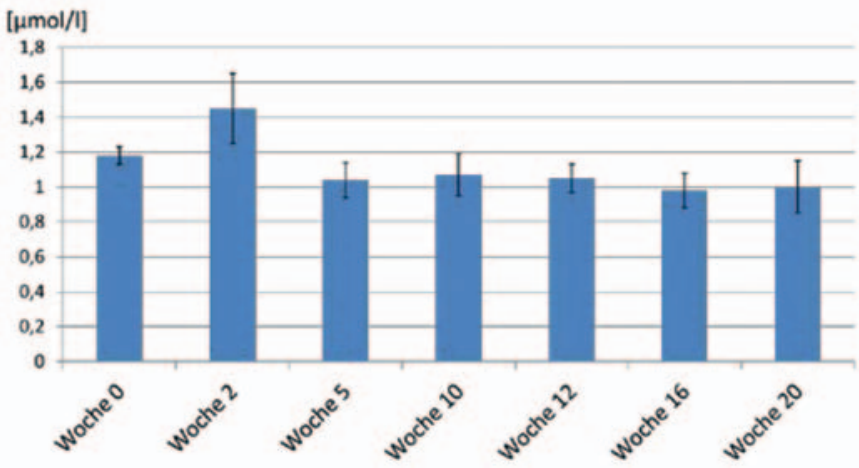

Abb. 5. Mittelwerte und deren Standardfehler von MDA im Blutplasma vor Saunabesuch sowie im Verlauf einer 20-wöchigen Saunaserie $(\mathrm{n}=10)$. Statistisch besteht ein signifikant abnehmender Trend (lineare Trendberechnung) $(\mathrm{p}<0,05)$.

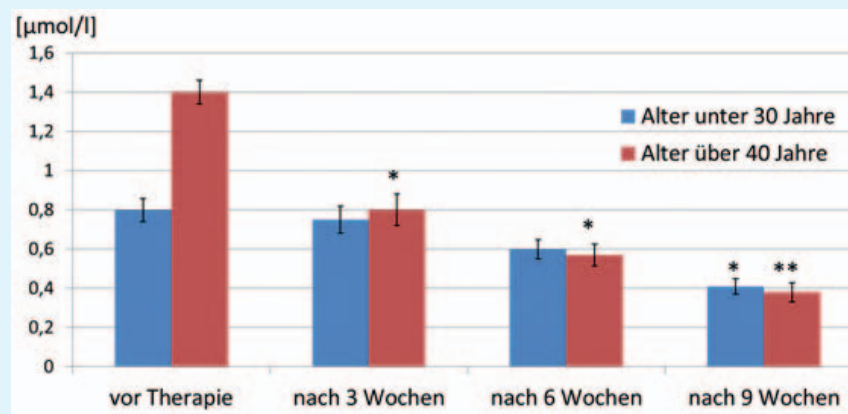

Abb. 6. MDA-Konzentration im Blutserum (Mittelwerte und Standardfehler) vor und während der Therapie mit Knoblauch (Allium sativum Linné) bei Probanden $(\mathrm{n}=25)$ unter $30(\mathrm{n}=12)$ und über 40 Jahre $(\mathrm{n}=13)$. Es wurden tägliche Dosen von $900 \mathrm{mg}$ eines getrockneten Knoblauch-Präparates Sapec ${ }^{\circledR} / \mathrm{Kwal}^{\circledR}$ der Firma Lichtwer, Berlin, Deutschland, eingesetzt. Das Präparat enthielt 1,3\% Allicin (Allicin-Release 0,6\%). Signifikante Unterschiede der jeweiligen MDA-Konzentration im Vergleich zu dem Wert vor Therapiebeginn bestehen im Wilcoxon-Test bei den Probanden über 40 Jahre nach 3 und 6 Wochen mit $\mathrm{p}<0,05\left(^{*}\right)$ und nach 9 Wochen sogar mit $\mathrm{p}<0,01\left(^{* *}\right)$; bei den Probanden unter 30 Jahren liegt der Wert nach 9 Wochen mit $\mathrm{p}<0,05\left(^{*}\right)$ signifikant unter dem Ausgangswert. und Adaptationen vorhanden wie nach ausschliesslichem Kaltreiz (Abb. 5).

\section{Phytotherapie}

«Ich habe viele Jahre hindurch zum grössten Teil mit Kräutern und weniger mit Wasser kuriert und dabei die schönsten Erfolge erzielt.» (Sebastian Kneipp)

Allgemein besitzen Phytopharmaka ein breites Indikationsspektrum. Auch hier war es naheliegend, Phytopharmaka, die sich durch eine besonders breite Indikation auszeichnen, näher auf ihre Wirkungen im Hinblick auf den Radikalmetabolismus zu untersuchen. Viele Phytopharmaka enthalten z.B. Flavonoide. Diese können als Radikalfänger wirken und sind häufig Komplexbildner für Metalle. Ausserdem enthalten viele Phytopharmaka weitere natürliche Antioxidantien. Im Unterschied zur Hydrotherapie, bei der es durch die Kaltreize zu einer dosierten Steigerung der Sauerstoffradikalbildung und damit zu aktiven Anpassungserscheinungen im Radikalmetabolismus analog zu einer aktiven Immunisierung kommt, wird bei der Phytotherapie ein anderes Wirkprinzip mit dem Ziel der Stärkung antioxidativer Schutzsysteme genutzt. Dieses besteht nicht in einem aktiven Training antioxidativer Mechanismen, sondern in einer passiven Zufuhr von Antioxidantien - vergleichbar mit einer passiven Immunisierung. Als weit verbreitetes Phytopharmakon wurde ein Knoblauch-Präparat untersucht [60-65]. Es konnte gezeigt werden, dass durch die Wirkung von Knoblauch (Allium sativum) die Konzentration eines wichtigen LPO-Metaboliten, des MDA, im Blutplasma signifikant gesenkt werden konnte. Die Untersuchungen erfolgten nach 3, 6 und 9 Wochen (Abb. 6).

Der MDA-reduzierende Effekt wurde in beiden untersuchten Altersgruppen gefunden. Besonders ausgeprägt war er jedoch bei älteren Probanden, bei denen die Ausgangswerte von MDA deutlich höher waren als bei den jüngeren Probanden. Der Einsatz mancher Phytopharmaka als prophylaktisches Mittel gegen vorschnelles Altern erscheint unter Berücksichtigung dieser Ergebnisse in einem neuen Licht. Parallel zur MDA-Senkung im Blutplasma fanden sich auch in den roten Blutzellen signifikante Veränderungen, und zwar in Form eines Anstiegs des Glutathiongehalts.

Eine ähnliche Wirkung, jedoch quantitativ geringer ausgeprägt, konnten wir auch für ein Präparat aus Ginkgo biloba nachweisen. Der antioxidative Effekt des Knoblauch-Präparates war jedoch deutlich grösser als der des Präparates aus G. biloba. Hier konnte zusätzlich ein Abfall der Enzymaktivität der Transaminase ASAT (SGOT) beobachtet werden. Dies ist insofern von Bedeutung, da die Leber ein Organ mit einer sehr hohen Radikalbildungsrate 
sowie - neben den Erythrozyten - mit der höchsten Kapazität der antioxidativen Schutzsysteme ist.

Die nützlichen Effekte von A. sativum einschliesslich seiner antioxidativen Wirkungen werden von Butt et al. [66] übersichtlich dargestellt. A. sativum befindet sich unter einer Reihe zahlreicher Phytopharmaka, die zur Prävention und Therapie von oxidativem Stress eingesetzt werden $[4,67-70]$.

\section{Ernährungstherapie}

«Wenn der Vater einer Erkrankung oft unbekannt ist, ist die Mutter immer die Ernährung.»

(Sebastian Kneipp)

Es gilt als gesichert, dass eine Vielzahl von Erkrankungen ernährungsabhängig ist. Neben einer Fehlernährung spielt insbesondere die Überernährung in den modernen Industrieländern eine grosse Rolle. Zwischen dieser theoretischen Erkenntnis und dem Umsetzen in die Praxis klafft jedoch eine grosse Lücke. So sollte eine gesunde und vollwertige Ernährung nicht nur möglichst naturbelassen und reich an Ballaststoffen sein, sondern sie beinhaltet ebenfalls die Zufuhr von Substanzen, die oft nur in pflanzlichen Nahrungsmitteln zu finden sind. Dazu zählen neben manchen Vitaminen auch Gewürze wie z.B. der Knoblauch. Dieser enthält z.B. Alliin, dessen SH-Gruppen als Bausteine für das Glutathion angesehen werden können, wodurch ein Zusammenhang mit dem antioxidativen Schutzsystem besteht.

Im Rahmen der Kneipp'schen Therapieverfahren hat das therapeutische Fasten als intensivste Form der Diätetik schon seit Langem eine grosse Rolle gespielt. Generell sollte der Kalorienrestriktion grosse Bedeutung beigemessen werden. Dabei wird das Fasten nicht nur zur Regulierung von Stoffwechselstörungen und zur Einleitung einer Therapie der Adipositas eingesetzt, sondern es besitzt ein sehr weites Indikationsspektrum. Bekannt sind die Wirkungen des Fastens z.B. auch beim Rheuma oder bei anderen entzündlichen Erkrankungen, bei denen freie Radikale eine grosse Rolle spielen. Immer wieder wird jedoch auch vor dem Fasten gewarnt. Einer der Gründe besteht darin, dass vermutet wird, dass es unter dem Fasten zu Mangelerscheinungen (z.B. an den lebensnotwendigen, antioxidativ wirkenden Vitaminen E und C) kommen kann.

Durch die Untersuchungen zusammen mit Jüttner [71] konnte gezeigt werden, dass nicht nur die Furcht vor einem Vitaminmangel bei dem derzeit üblichen Fastenregime wir bevorzugen das Fasten in Anlehnung an Buchinger unbegründet war: Die Vitamine $\mathrm{E}$ und $\mathrm{C}$ blieben im Fastenverlauf konstant. Während des Buchinger-Fastens kommt es zu einem signifikanten und kontinuierlichen Abfall von MDA als einem wichtigen Reaktionsprodukt

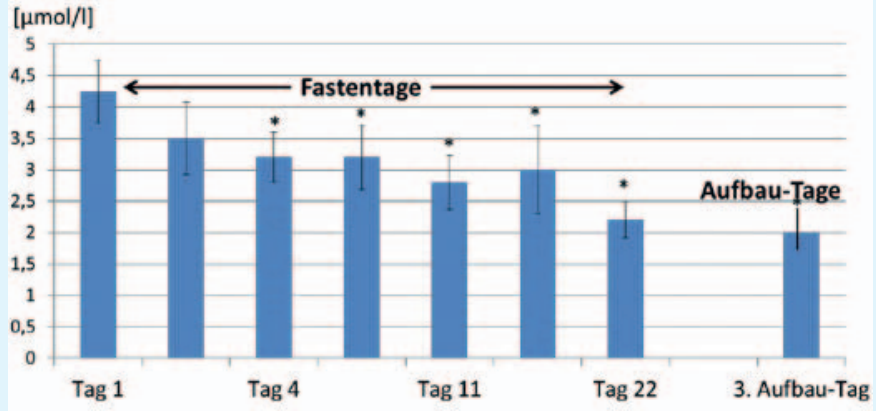

Abb. 7. Abfall der MDA-Konzentration im Blutserum bei einer Gruppe von Patienten $(\mathrm{n}=16)$, die gefastet hat (Buchinger-Fasten). Dargestellt sind die Mittelwerte und Standardfehler der 16 Personen. $\mathrm{Ab}$ dem 4 . Fastentag ist der Unterschied gegenüber den Ausgangswerten statistisch $\left({ }^{*}\right)$ signifikant (Wilcoxon-Test; $\mathrm{p}<0,05$ ). Dieser Unterschied bleibt auch zumindest während der Aufbautage in der Nachfasten-Periode erhalten. Die höheren Ausgangswerte als bei den Sauna-Probanden erklären sich durch die andere Ausgangssituation, d.h. die Krankheitsbilder der Fasten-Patienten im Sinne eines metabolischen Syndroms.

von Radikalen mit ungesättigten Fettsäuren. Es konnte vermutet werden, dass LPO-Prozesse durch den Substanzverlust an Gesamtlipiden im Serum reduziert werden. Die Belastung mit Reaktionsprodukten von freien Radikalen des Organismus wird somit durch das Fasten geringer (Abb. 7). Dies könnte einer der Gründe für die zusätzlich beobachtete Abnahme von Entzündungsaktivitäten im Organismus sein. Das Konzept des therapeutischen Fastens ordnet sich somit in die Kneipp'schen Therapieverfahren und in die von uns postulierte allgemeine Beeinflussung des Radikalmetabolismus ein. Aktuelle Übersichten über das Fasten sind selten [72]. Übersichten über den Nutzen von Antioxidantien in der normalen Ernährung sind hingegen ein häufiges Thema der angewandten medizinischen Forschung [73, 74].

\section{Bewegungstherapie und Ordnungstherapie}

"Zu Fuss will heute niemand mehr gehen und doch hat das Gehen im Freien einen sehr grossen Einfluss auf unsere Gesundheit.»

«Um gesund zu bleiben, muss der Mensch sich bewegen, schwitzen.»

"Zu den Wasseranwendungen gehört auch die Bewegung, beide greifen ineinander.»

"Kaum irgendein Umstand kann schädlicher auf die Gesundheit wirken als die Lebensweise unserer Tage: Ein fieberhaftes Hasten und Drängen aller im Kampfe um Erwerb und sichere Existenz.»

"Oft konnte ich den kranken Menschen erst helfen, nachdem ich Ordnung in ihre Seelen gebracht hatte.» (Sebastian Kneipp) 
Unumstritten ist weiter die Tatsache, dass ein Bewegungsmangel zum Risikofaktor werden kann. Andererseits hat ein dosiertes körperliches Training günstige Auswirkungen auf den Gesundheitszustand des menschlichen Organismus. Der Sauerstoffverbrauch wird durch körperliches Training stark erhöht, was eine vermehrte Bildung von Sauerstoffradikalen auslöst [75-77]. Dabei spielen auch die Katecholamine eine wesentliche Rolle. Der Abbau von Katecholaminen ist darüber hinaus eine wichtige Quelle für die Bildung von Sauerstoffradikalen (n1). Die Arbeit von Göhler et al. [78] zeigt den ausgeprägten Anstieg von Adrenalin, Noradrenalin und auch Dopamin im Blutplasma nach einer physischen Belastung, was mit einer verstärkten Bildung von ROS durch Autoxidation der Katecholamine einhergeht. Die Katecholamine Adrenalin und Noradrenalin sind der Inbegriff von Stressinduktion, Anstrengung und intensiver konzentrierter Arbeit. Stressabbau führt zu niedrigerer Katecholamin-Ausschüttung. Stressabbau und Ordnungstherapie können somit die Bildung von ROS reduzieren und die Antioxidantien stärken. Unter einer 16-tägigen Fastenperiode kommt es nicht nur zu einem deutlichen Absinken der Ausgangswerte von Adrenalin und Noradrenalin, sondern auch der Anstieg der Katecholamin-Konzentrationen unter physischer Belastung fällt vergleichsweise geringer aus. Die Senkung der sympathikoadrenalen Aktivität geht eindeutig mit einer verringerten radikalischen Belastung des Organismus einher - messbar an verringerten Konzentrationen von LPO-Produkten und an einer «Entlastung» des Glutathionsystems. Dies entspricht auch dem allgemeinen Kenntnisstand, dass es im untrainierten Organismus bei körperlicher Belastung zu einer besonders starken Erhöhung der GSSG-Konzentration und des GSSG-Glutathion-Quotienten in Leber, Herz, Skelettmuskulatur und Blutplasma kommt, jedoch solche Erhöhungen bei trainierten Probanden nicht oder nur in geringem Umfang auftreten. Weiterhin fanden sich nach dem Fasten erhöhte Konzentrationen verschiedener Antioxidantien im Blutplasma. Nur am Rande sei erwähnt, dass die Abnahme der sympathikoadrenalen Aktivität unter dem therapeutischen Fasten einem ordnungstherapeutischen Ansatz entspricht und im Sinne einer positiven Reaktion zu werten ist.

Ähnlich wie bei der Hydrotherapie kann auch für die Bewegungstherapie davon ausgegangen werden, dass die gering dosierte wiederholte körperliche Belastung einer milden oxidativen Belastung entspricht, die keine funktionellen oder morphologischen Zellschäden hervorrufen kann, sondern zu sinnvollen Anpassungserscheinungen führt. Man kann durch regelmässiges körperliches Training mit relativ gering dosierter Einzelbelastung eine Zunahme der antioxidativen Kapazität des Organismus erzielen.

\section{Oxidativer Stress und Kneipp-Therapie: Aufklärung molekularer Feinmechanismen eines populären Verfahrens - Schlussfolgerung, Ausblick, kritische Diskussion der Hypothese}

Erstmals wurde 1991 die Vermutung geäussert, dass das Regimen refrigerans als ein oxidativer Stress angesehen werden kann [58]. Zwar ist ein Kaltreiz intensiv genug, um erwünschte Anpassungen hervorzurufen, jedoch werden dabei keine Schäden der Muskulatur oder anderer Organe beobachtet. Diese Untersuchungen haben sich in den letzten Jahren durch unsere Befunde bestätigt. Dies wird als ein neuer Wirkmechanismus der Abhärtung durch wiederholte Kaltreize auf biochemischer Ebene interpretiert. Mittlerweile sind die Auffassungen in verschiedener Richtung detailliert untersucht und publiziert worden - durch uns und durch andere Gruppen - und auch bei anderen Spezies als dem Menschen bestätigt. Man kann also mit regelmässig wiederholten milden Kaltreizen den oxidativen Stress mildern und antioxidative Prävention betreiben.

Die vorliegende Übersicht demonstriert weiterhin, dass sich das Prinzip einer Beeinflussung der Bildung und Bewältigung von ROS quer durch alle Kneipp'schen Therapieverfahren zieht. Die punktuell durch uns bearbeiteten Detailthemen für die einzelnen Säulen der Kneipp-Therapie stützen die Hypothese. Die Bearbeitung weiterer Unterthemen in der internationalen Literatur gibt zahlreiche Hinweise auf die Möglichkeit, die antioxidativen Schutzsysteme für den Gewinn von Gesundheit und Leistungsfähigkeit zu stärken (siehe Zitate zur Phytotherapie und zur Ernährung).

Die modernen Studien mehrerer Forschungsgruppen, vor allem von Jose Viña, zu Bewegung und Training belegen sogar, dass mildes, regelmässig wiederholtes Training zu einem stärkeren antioxidativen Schutz beiträgt als eine hoch dosierte Supplementation von Antioxidantien. Aus den Ergebnissen von Viña et al. [79-83] kann man sogar schlussfolgern, dass Bewegungstherapie das beste Antioxidans sein könnte.

Wir sind gewiss, dass die Aufdeckung weiterer molekularer Wirkmechanismen mit modernsten analytischen Methoden der Genomik, Proteomik und Metabolomik zu einer weiteren Akzeptanz der von Kneipp eingeführten alten und doch so aktuellen und wirksamen Verfahren beitragen kann und wird. Der Fehler, den wir machen können, besteht darin, dass wir die Prinzipien der Milde, Regelmässigkeit und Ganzheitlichkeit verletzen - oder dass wir die Naturheilverfahren missachten und ignorieren. Das sinnvolle Zusammenspiel von Schulmedizin und Komplementärmedizin scheint von höchster medizinischer Aktualität und Relevanz für die Erhaltung und Wiederherstellung von Gesundheit, Leistungsfähigkeit und Lebensqualität. 


\section{Disclosure Statement}

Es liegen keine Interessenkonflikte durch Sponsoring oder Unterstützung

durch Industrie oder andere Institutionen vor.

\section{Literatur}

1 Siems W, Krämer K, Grune T: Oxidativer Stress und Pharmaka. Eschborn, Govi, 2005, pp 1-279.

$>2$ Spickett CM, Wiswedel I, Siems W, Zarkovic K, Zarkovic N: Advances in methods for the determination of biologically relevant lipid peroxidation products. Free Radic Res 2010;44:1172-1202.

$\checkmark 3$ Negre-Salvayre A, Auge N, Ayala V, et al. Pathological aspects of lipid peroxidation. Free Radic Res 2010;44:1125-1171.

4 Poli G, Schaur RJ, Siems W, Leonarduzzi G: 4-Hydroxynonenal: a membrane lipid oxidation product of medicinal interest. Med Res Rev 2008;28:569-631.

$\checkmark 5$ Gueraud F, Atalay M, Bresgen N, Dipak Gasparovic A, Eckl PM, Huc L, Jouanin I, Siems WG, Uchida K: Chemistry and biochemistry of lipid peroxidation. Free Radic Res 2010;44:1098-1124.

6 Zarkovic K, Juric G, Waeg G, Kolenc D, Zarkovic $\mathrm{N}$ : Immunohistochemical appearance of HNE-protein conjugates in human astrocytomas. Biofactors 2005;24:33-40.

7 Ray PDM, Huang BW, Tsuji Y: Reactive oxygen species (ROS) homeostasis and redox regulation in cellular signaling. Cell Signal 2012;24:981-990.

8 Grune T, Siems WG, Zollner H, Esterbauer H: Metabolism of 4-hydroxynonenal, a cytotoxic lipid peroxidation product, in Ehrlich mouse ascites cells at different proliferation stages. Cancer Res 1994:54:5231-5235.

$\checkmark 9$ Maulik SK, Kumar S: Oxidative stress and cardiac hypertrophy: a review. Toxicol Mech Methods 2012;22:359-366.

$>10$ Puertollano MA, Puertollano E, de Cienfuegos GA, de Pablo MA: Dietary antioxidants: immunity and host defense. Curr Top Med Chem 2011;14:1752-1766.

-11 Schaffer SW, Jong CJ, Mozaffari M: Role of oxidative stress in diabetes-mediated vascular dysfunction: unifying hypothesis of diabetes revisited. Vascul Pharmacol 2012;57:139-149.

-12 Winyard PG, Ryan B, Eggleton P, Nissim A, Taylor E, Lo Faro ML, Burkholz T, Szabó-Taylor KE, Fox B, Viner N, Haigh RC, Benjamin N, Jones AM, Whiteman M: Measurement and meaning of markers of reactive species of oxygen, nitrogen and sulfur in healthy human subjects and patients with inflammatory joint disease. Biochem Soc Trans 2011;39:12261232.

$\checkmark 13$ Chen SD, Yang DI, Lin TK, Shaw FZ, Liou CW, Chuang YC: Roles of oxidative stress, apoptosis, PGC-1alpha and mitochondrial biogenesis in cerebral ischemia. Int J Mol Sci 2011;21:7199-7215.

14 Castellani RJ, Moreira PI, Perry G, Zhu X: The role of iron as a mediator of oxidative stress in Alzheimer disease. Biofactors 2012;38: 133-138.
15 Fletcher AE: Free radicals, antioxidants and eye diseases: evidence from epidemiological studies on cataract and age-related macular degeneration. Ophthalmic Res 2010;44:191198.

16 Wiswedel I, Peter D, Gardemann A, Carluccio F, Hampl H, Siems W: Serum concentrations of $\mathrm{F}_{2}$-isoprostanes and 4-hydroxynonenal in hemodialysis patients with endstage renal disease in relation to inflammation and renal anemia. Biofactor Insights 2008;3: $1-10$.

17 Siems W, Carluccio F, Radenkovic S, Grune T, Hampl H: Oxidative stress in renal anemia of hemodialysis patients is mitigated by epoetin treatment. Kidney Blood Press Res 2005;28:295-301

18 Siems W, Quast S, Carluccio F, Wiswedel I, Hirsch D, Augustin W, Kraemer K, Hampl H, Sommerburg O: Oxidative stress in cardiorenal anemia-syndrome: correlations and therapeutic possibilities. Clin Nephrol 2003; 60(suppl 1):S22-S30.

19 Siems WG, Brenke R, Beier A, Grune T: Oxidative stress in chronic lymphedema. QJM 2002;95:803-809.

20 Siems W, Brenke R: Chronische Lymphödeme umfassend behandeln. Pharmazeut $\mathrm{Z}$ 2004;149:3224-3230.

21 Brenke R, Siems W: Medikamentöse und antioxidative Therapie beim Lymphödem Pro und Kontra. Lymph Forsch 2005;9:8691.

22 Siems W, Brenke R, Wiswedel I, Sommerburg O, Eckl P, Bresgen N: Erhöhte Oxysterol-Spiegel beim chronischen Lymphödem. Lymph Forsch 2009;13:29-33.

23 Hegde ML, Mantha AK, Hazra TK, Bhakat KK, Mitra S, Szczesny B: Oxidative genome damage and its repair: implications in aging and neurodegenerative diseases. Mech Ageing Dev 2012;133:157-168.

24 Gil L, Siems W, Mazurek B, Gross J, Voss P, Grune T: Age-associated analysis of oxidative stress parameters in human plasma and erythrocytes. Free Radic Res 2006;40:495-505.

25 Siems W, Voss P: Clinical oxidation parameters of aging. Free Radic Res 2006;40:1339-1349.

26 Breusing N, Arndt J, Voss P, Bresgen N, Wiswedel I, Gardemann A, Siems W, Grune T: Inverse correlation of protein oxidation and proteasome activity in liver and lung. Mech Ageing Dev 2009;130:748-753.

27 Sohal RS, Orr WC: The redox stress hypothesis of aging. Free Radic Biol Med 2012;52:539555 .

28 Calabrese V, Cornelius C, Cuzzocrea S, Iavicoli I, Rizzarelli E, Calabrese EJ: Hormesis, cellular stress response and vitagenes as critical determinants in aging and longevity. Mol Aspects Med 2011;32:279-304
29 Grune T, Schröder P, Siems W: Antioxidative Verteidigung - Niedermolekulare Abwehrtruppe. Pharmazeut Z 2005;150:1410-1415.

30 Siems WG, Sommerburg O, Mayer H, Grune T: Die wichtigsten Radikalquellen im menschlichen Organismus - Pharmakologische Ansatzpunkte. Pharmazeut Z 1998;143:15151527.

31 Merker K, Siems W, Grune T: Oxidativer Stress lässt Zellen schneller altern - Reaktive Sauerstoffspezies: Lebenslange Attacke tötet Zellen. Pharmazeut Z 2002;44:4208-4214.

32 Schrödinger E: Was ist Leben? Die lebende Zelle mit den Augen des Physikers betrachtet, ed 3. München, Piper, 1989, p 42.

33 Siems W, Voss P, Bresgen N, Eckl PM, Grune T: HNE (4-hydroxynonenal) metabolism and its age dependency. J Med Sci 2009;29:7-10.

34 Siems W, Grune T: Lipid peroxidation measurements - methodological approaches and clinical importance; in Grune $\mathrm{T}$ (ed): Free Radicals and Diseases: Gene Expression, Cellular Metabolism and Pathophysiology. NATO Science Series. Amsterdam, IOS, 2005, pp 11-21.

35 Siems WG, Krämer K, Grune T: Zur Chemie und Biologie des Glutathionsystems: ein Überblick. Pharmazeut Z 1996;141:4239-4248.

36 Siems WG, Krämer K, Grune T: Störungen im Glutathionsystem und klinische Konsequenzen. Pharmazeut Z 1996;141:4343-4345.

37 Siems WG, Zollner H, Grune T, Esterbauer H: Metabolic fate of 4-hydroxynonenal in hepatocytes: 1,4-Dihydroxynonene is not the main product. J Lipid Res 1997;38:612-622.

38 Grune T, Siems WG, Petras T: Identification of metabolic pathways of the lipid peroxidation product 4-hydroxynonenal in in situ perfused rat kidney. J Lipid Res 1997;38:1660-1665.

39 Siems WG, Pimenov AM, Esterbauer $\mathrm{H}$, Grune T: Metabolism of 4-hydroxynonenal, a cytotoxic lipid peroxidation product, in thymocytes as an effective secondary antioxidative defense mechanism. J Biochem 1998; 123:534-539.

40 Siems WG, Grune T: HNE metabolism as secondary antioxidative system. Rev Farmácia Bioquímica Sao Paulo 1998;34(suppl 1):119.

41 Siems W, Grune T: Intracellular metabolism of 4-hydroxynonenal. Mol Aspects Med 2003; 24:167-175.

42 Siems W: HNE metabolism - part of the secondary antioxidative defense system; in Kumar A (ed): International Conference on 'Free Radicals und Natural Products in Health' FRNPH and 'Seventh Annual Meeting of the Society of Free Radical Research-India' Proceedings. New Delhi, University Press Jaipur, 2009, pp 1-10.

43 Brenke R, Bühring M: Abhärtung durch Hydrotherapie? Z Allg Med 1997;73:18-23. 
44 Brenke R, Siems W: Das Buch vom Winterschwimmen. Gesund und fit durch Abhärtung. Husum, Husum Druck- und Verlagsgesellschaft, 1996, pp 1-128.

45 Brenke R: Baden im eisigen Wasser - über den gesundheitlichen Wert einer Extremform der Abhärtung. Saunabetrieb Bäderpraxis 1997; 4:20-26.

46 Brenke R, Siems W: Whole body cold stimulus during winter swimming: an oxydative stress; in Conradi E (ed): Physical Medicine and Rehabilitation. Trends and New Approaches in Physical Medicine and Rehabilitation. Berlin, ABC, 1992, p 317.

47 Siems W, Brenke R, Grune T, Conradi E: Regulation of generation and removal of oxygen free radicals as result of hydrotherapy, phytotherapy and exercise; in Conradi E (ed) Physical Medicine and Rehabilitation. Trends and New Approaches in Physical Medicine and Rehabilitation. Berlin, ABC, 1992, p 22.

48 Brenke R, Siems W, Maaß R: Abhärtung durch Kaltreize unterschiedlicher Intensität: Wirkungen auf den Purin- und Radikalmetabolismus. WMW 1994;144:66-68.

49 Brenke R, Siems WG, Maaß R, van Kujik FJGM: Konzentration von Harnsäure und Glutathion als wichtige natürliche Antioxidantien beim Winterschwimmen - neue Aspekte zu molekularen Grundlagen von Abhärtungsmechanismen. Akt Ernährungsmed 1994;19:56.

50 Siems WG, van Kujik FJGM, Maass R, Grune T, Brenke R: Uric acid, glutathione and vitamin $\mathrm{C}$ levels during short-term whole body cold exposure; in Xin W, Packer L, Gu F (eds): Natural Antioxidants - Molecular Mechanisms and Health Effects. Beijing, Acad. Sinica, 1995 p 320 .

51 Siems W, Brenke R: Changes in the glutathione system of erythrocytes due to enhanced formation of oxygen free radicals during shortterm whole body cold stimulus. Arctic Med Res 1992;51:3-9.

-52 Siems WG, van Kujik FJGM, Maass R, Brenke R: Uric acid and glutathione levels during short-term whole body cold exposure. Free Radic Biol Med 1994;16:299-305.

53 Siems W, Brenke R, Sommerburg O, Grune T: Improved antioxidative protection in winterswimmers: adaptation to repeated oxidative stress; in Grune T, Davies K, Flohé L, Sies H (eds): Antioxidants, Adaptation, Aging, SFRR Europe Summer Meeting. Dresden 1999, p 63.

54 Martarelli D, Cocchioni M, Scuri S, Spataro A, Pompei P: Cold exposure increases exerciseinduced oxidative stress. J Sports Med Phys Fitness 2011;51:299-304.

55 Tseng YC, Chen RD, Lucassen M, Schmidt MM, Dringen R, Abele D, Hwang PP: Exploring uncoupling proteins and antioxidant mechanisms under acute cold exposure in brains of fish. PLoS One 2011;6:e18180.
56 Blagojevic DP, Grubor-Lajsic GN, Spasic MB: Cold defence responses: the role of oxidative stress. Front Biosci (Schol Ed) 2011;3:416-427.

57 Papke M, Brenke R, Siems W: Hydrotherapie; in Siems W, Bremer A (Hrsg): Physiotherapie - Das Ausbildungsscript. Band II: Behandlungstechniken. Auerbach, Wissenschaftliche Scripte, 2009, pp 99-138.

58 Brenke R, Siems W: Eine biochemische Hypothese zur Erklärung der Wirkung von Kaltreizen: Das regimen refrigerans - ein dosierter oxydativer Streß? Kneipparzt 1991;1:21-24.

59 Conradi E, Brenke R, Grune T, Grünberger P, Kästner A: Beeinflussung des Radikalstoffwechsels durch Sauna und kurzzeitige Abkühlung. Int Sauna Arch 1994;11:55-59.

60 Brenke R, Siems W, Scherat T, Conradi E: Antioxydative Effekte ausgewählter Phytopharmaka (Abstract). Phys Rehab Kur Med 1994;4:147.

61 Scherat T, Siems WG, Brenke R, Grune T, Jakstadt M, Conradi E: Influence of vitamin E, Allium sativum and Ginkgo biloba extract Egb 761 on malondialdehyde level in human blood plasma; in Krinsky NI, Sies H (eds): Antioxydant Vitamins and Beta-Carotene in Disease Prevention. Berlin, Society for Free Radical Research, 1994, p 49.

62 Siems WG, Grune T, Scherat T, Behrend H, Conradi E, Brenke R: Influence of Allium sativum Linne on oxidative stress status a clinical investigation; in Xin W, Packer L, Gu F (eds): Natural Antioxidants - Molecular Mechanisms and Health Effects. Beijing, Acad. Sinica, 1995, p 179.

63 Siems WG, Scherat T, Behrend H, Brenke R, Jakstadt M, Conradi E, Grune T: Influence of Allium sativum Linne on oxidative stress status - a clinical investigation. Chapter 20; in Packer L, Traber MG, Xin W (eds): Natural Antioxidants: Molecular Mechanisms and Health Effects. New York, AOCS, 1996, pp 188-195.

64 Grune T, Scherat T, Behrendt H, Conradi E, Brenke R, Siems W: Influence of Allium sativum Linne on oxidative stress status - a clinical investigation. Phytomedicine 1996;2:205-207.

65 Scherat T, Siems WG, Brenke R, Behrend H, Jakstadt M, Conradi E, Grune T: Influence of a garlic extract on oxidative stress parameters in human. International Conference on $\mathrm{Na}$ tural Antioxidants and Lipid Peroxidation in Artherosclerosis and Cancer (ALPAC), Helsinki 1995. Conf. Proc. Royal Chemical Society 1996, pp 286-289.

66 Butt MS, Sultan MT, Butt MS, Iqbal J: Garlic: nature's protection against physiological threats. Crit Rev Food Sci Nutr 2009;49:538551.

67 Kelsey NA, Wilkins HM, Linseman DA: Neutraceutical antioxidants as novel neuroprotective agents. Molecules 2010;15:7792-7814.

68 Masterjohn C, Bruno RS: Therapeutic potential of green tea in nonalcoholic fatty liver disease. Nutr Rev 2012;70:41-45.
69 Wilkinson JT, Fraunfelder FW: Use of herbal medicines and nutritional supplements in ocular disorders: an evidence-based review. Drugs 2011;71:2421-2423.

70 Javed S, Kohli K, Ali M: Reassessing bioavailability of silymarin. Altern Med Rev 2011; 16:239-249.

71 Jüttner U: Untersuchungen zum oxidativen Gleichgewicht im therapeutischen Fasten bei stoffwechselgesunden Patienten. Medizinische Dissertation. Berlin, Humboldt-Universität, 1996.

72 Burke L: Fasting and recovery from exercise. Br J Sports Med 2010;44:502-508.

73 Calabrese V, Cornelius C, Trovato A, Cavallaro M, Mancuso C, Di Rienzo L, Condorelli D, De Lorenzo A, Calabrese EJ: The hermetic role of dietary antioxidants in free radical-related diseases. Curr Pharm Des 2010;16:877-883.

74 Hankey GJ: Nutrition and the risk of stroke. Lancet Neurol 2012;11:66-81.

75 Brenke R, Siems W: Physisches Training ein oxidativer Stress; in Conradi E, Brenke R (Hrsg): Bewegungstherapie: Grundlagen, Ergebnisse, Trends. Berlin, Ullstein \& Mosby, 1993, pp 53-57.

76 Siems W, Brenke R, Grune T, Conradi E: Regulation and removal of oxygen free radicals as result of hydrotherapy, phytotherapy and exercise. XIth World Congress of the International Federation of Physical Medicine and Rehabilitation, 1992. Abstracts, p 22.

77 Siems W, Brenke R, Grünberger P, Conradi E: Bedeutung einer verstärkten Bildung freier Radikale bei Hydro- und Bewegungstherapie. Phys Rehab Kur Med 1994;159:4.

78 Göhler L, Hahnemann T, Michael N, Oehme P, Steglich H-D, Conradi E, Grune T, Siems WG: Reduction of plasma catecholamines in humans during clinically controlled severe underfeeding. Prev Med 2000;30:95-102.

79 Gomez-Cabrera MC, Viña J, Ji LL: Interplay of oxidants and antioxidants during exercise: implications for muscle health. Phys Sportsmed 2009;37:116-123.

80 Ji LL, Gomez-Cabrera MC, Viña J: Role of free radicals and antioxidant signaling in skeletal muscle health and pathology. Infect Disord Drug Targets 2009;9:428-444.

81 Gomez-Cabrera MC, Domenech E, Viña J: Moderate exercise is an antioxidant: upregulation of antioxidant genes by training. Free Radic Biol Med 2008;44:126-131.

82 Viña J, Gomez-Cabrera MC, Lloret A, Marquez R, Miñana JB, Pallardó FV, Sastre J: Free radicals in exhaustive physical exercise: mechanism of production, and protection by antioxidants. IUBMB Life 2000;50:271-277.

83 Ji LL, Gomez-Cabrera MC, Viña J: Exercise and hormesis: activation of cellular antioxidant signaling pathway. Ann N Y Acad Sci 2006;1087:425-435. 